\title{
A Digital Emission-Line Survey of the Small Magellanic Cloud
}

\author{
P. Frank Winkler, Yasser Rathore \\ Middlebury College, Middlebury, VT 05753, USA \\ R. Chris Smith \\ University of Michigan, Ann Arbor, MI 48109, USA
}

\begin{abstract}
We present results on the SMC from the first full season of the Michigan/CTIO Magellanic Cloud Emission-Line Survey, being carried out from CTIO. Images are being obtained in $\mathrm{H} \alpha$, [S II] $\lambda \lambda$ 6717, 6731, and [O III] $\lambda 5007$, plus red and green continuum bands for star subtraction. Data from the 1996-97 season have been assembled into large mosaic images which reveal the rich variety of nebulosity in the SMC in unprecedented detail. These are providing definitive samples of the active, occasionally violent, ISM on scales including superbubbles, wind-blown bubbles, supernova remnants, $\mathrm{H}$ II regions, and planetary nebulae.
\end{abstract}

The Michigan/CTIO Magellanic Cloud Emission-Line Survey (MCELS) will image virtually all of both the Clouds at the wavelengths of $\mathrm{H} \alpha$, [S II], and [O III]. (See the paper by R.C. Smith in this volume for further details on the MCELS.) During the 1996-97 season at CTIO, a total of 34 overlapping 1.1 square fields in the SMC were observed from the $0.6 / 0.9 \mathrm{~m}$ Curtis Schmidt and 2K STIS CCD. Conditions were excellent; 14 of the 18 separate nights of observations were photometric.

The survey fields cover virtually the entire SMC, but for this presentation we concentrate on the $2.7 \times 2^{\circ} .1$ central region. The data for this region have been assembled into mosaic images on a standard coordinate system at a scale of $2^{\prime \prime} \cdot 0$ pixel $^{-1}$. The continuum images have been scaled and subtracted from those in the emission lines, in order to remove most of the stellar contribution and reveal faint nebulosity. In Figure 1 we show the continuum-subtracted $\mathrm{H} \alpha$ mosaic. The rms noise level corresponds to $2.5 \times 10^{-17} \mathrm{ergs} \mathrm{cm}^{-2} \mathrm{~s}^{-1} \operatorname{arcsec}^{-2}$; diffuse features down to almost this level are readily apparent. The sensitivity is comparable in the $[\mathrm{SII}]$ and $[\mathrm{O} \mathrm{III]} \mathrm{bands.}$

Through comparison of the emission in different bands we can classify many of the emission features; e.g., discrete structures with $[\mathrm{S} \mathrm{II}] / \mathrm{H} \alpha$ flux ratio $>0.4$ are probably supernova remnants, while marginally resolved objects with high $[\mathrm{O}$ III] $/ \mathrm{H} \alpha$ and weak [S II] are almost certainly planetary nebulae. All but one of the previously cataloged SNRs are apparent, and numerous new ones are seen as well.

Acknowledgments. This work has been supported in part by the NSF through grants AST-9618465 and AST-9530747, with additional support from the W.M. Keck Foundation through the Keck Northeast Astronomy Consortium. 
Winkler, Rathore and Smith

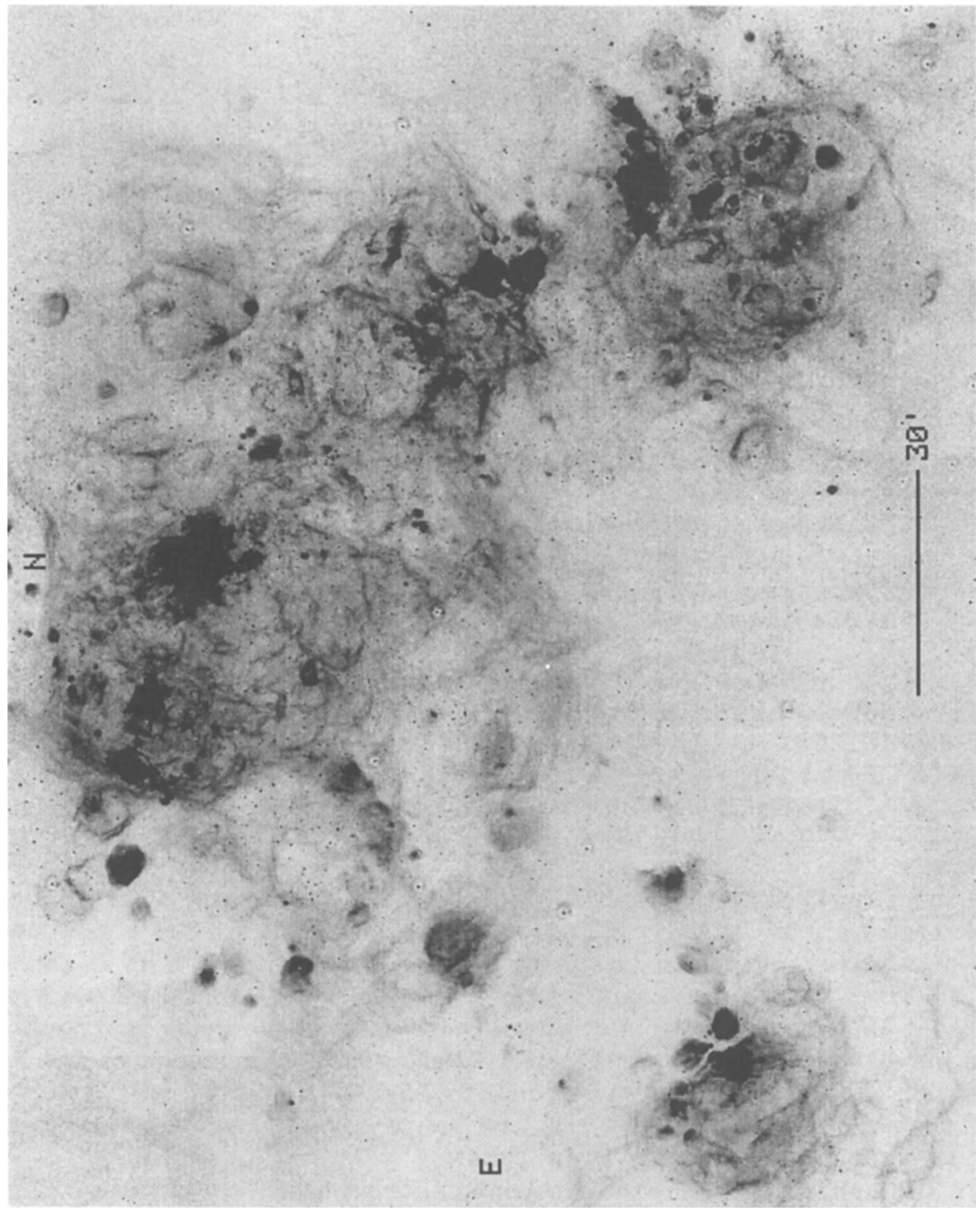

Figure 1. This continuum-subtracted $\mathrm{H} \alpha$ mosaic image of the central region of the SMC reveals a rich variety of structures on a wide range of scales. This image covers a $2.7 \times 2.1$ region at a scale of $2^{\prime \prime} \cdot 0$ pixel $^{-1}$; the full survey covers 4 times this area-essentially the entire SMC. 